\title{
Powerful and Novel Tumour Detection in Brain MRI Images Employing Hybrid Computational Techniques
}

\author{
K Lakshmi Narayanan ${ }^{\text {a,1 }}$, R Niranjana ${ }^{\text {a }}$ E Francy Irudaya Rani ${ }^{\text {a }, ~ N ~ S u b b u l a k s h m i ~}{ }^{\text {a }}$, \\ R Santhana Krishnan ${ }^{\mathrm{b}}$ \\ ${ }^{a}$ Assistant Professor, Francis Xavier Engineering College, Tirunelveli, India \\ ${ }^{b}$ Assistant Professor, SCAD College of Engineering and Technology, Tirunelveli, India
}

\begin{abstract}
Brain tumour detection is an evergreen topic to attract attention in the examination field of Information Technology innovation with biomedical designing, in view of the gigantic need of proficient and viable strategy for assessment of enormous measure of information. Image segmentation is considered as one of the most vital systems for visualizing tissues in an individual. To robotize image segmentation, we have proposed a calculation to get global optimal thresholding esteem for a specific brain MRI image, utilizing OTSU+Sauvola binarization strategy. The fundamental reason for feature collection is to diminish the quantity of structures utilized in classification while keeping up satisfactory classification exactness. One of the most extra-customary procedures applied for feature extraction is Discrete Wavelet Transform (DWT). Adequately it anticipates the estimation space on a plane to such an extent that the fluctuation of the information is ideally protected. We propose a justifiable model for brain tumours discovery and classification i.e., to classify whether the tumour is benign or malignant, utilizing SVM classification. SVM utilized here deals with basic hazard minimization to group the images for the tumour extraction, and a Graphical User Interface is created for the tumour classification operation, using the MATLAB platform.
\end{abstract}

Keywords. Brain tumour, MRI image, Discrete Wavelet Transform, SVM

\section{Introduction}

Normal specific forms of brain tumour include gliomas and meningiomas. Be that as it may, whether they are helpful or negative is the basic category within them. MRI is an expert technique in imaging for identifying brain tumours. In either case, simple manual analysis by people of these MRI images cannot be sufficient either to reliably assess a tumour's closeness or to classify the suspected tumour as benevolent or dangerous. This could spur human errors. To maintain a strategic distance from such an erroneous inquiry and to provide a reliable procedure to break down the brain pictures naturally and to determine specifically whether or not there is a tumour and also trained algorithms are also used to determine whether a tumour is dangerous or not. Premalignant tumour is a cancer stage where cancer can be treated while ignorance can lead to cancer. The disease is known as malignant tumour.

${ }^{1}$ K.Lakshmi Narayanan, Assistant Professor, Francis Xavier Engineering College, Tirunelveli, India; Email: drkln86@gmail.com. 
When it occurs, it starts to expand around neighbouring tissues after a particular period. Malignant tumours develop very rapidly and can cause a person's death. Early detection of malignant tumour can be of great benefit to the patient. That is why malignant tumour is immediately identified and marked a new way of achieving optimum precision is being investigated.

\section{Methodology}

\subsection{Pre-Processing}

The overall block diagram that depicts the process flow in the proposed technique is shown in Figure 1.

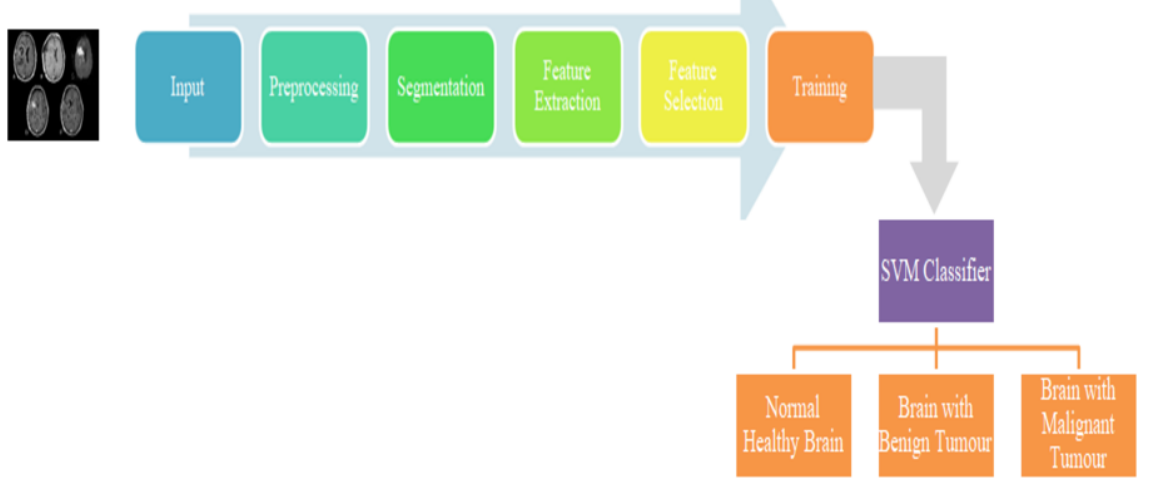

Figure 1. Process flow of proposed system

MRI images by large are subjected to alterations by a distortion of the bias field, which makes the asset of the same tissues vary throughout the picture. In order to make right this situation N4ITK was employed [1]. Whatsoever, this did not seem to justify the fact that intensity dispersal of a tissue type is a worthy match to intensity that various volunteers proved to show for similar series of MRI images [2]. In addition, it may also swerve from expected results, even if the patient is subjected to scans using the same imaging modality at different times or only when abnormality is detected[3]. So intensity normalization tactic advised by Nyúletal[3] was employed and analysed on each series in order to improve the sudden changes in intensity ranges which would be more distinct among different numerous cases. Through this form of intensity normalization, a sequence of depth symbols can be imbibed from training sets of images and are selected as defined in [4], for each MRI sequence.

\subsection{Segmentation \& Feature Extraction}

This paper suggests a more traditional approach which is a combination of two strategies, the binarization strategy OTSU+Sauvola. The Otsu method of segmentation is now well known for its capabilities in segmentation. But when combined with Otsu the Sauvola binarization results in an excellent segmented image. In particular, this combination works amazingly well for non-uniform context, as is the case with brain MRI image. It will detect the melanoma but it also takes into account a lot of 
distracting surrounding. Several image processing applications deploy Otsu's system to perform histogram technique based thresholding or perform binary thresholding [5]. The algorithm presented takes into account that the image accepts bi-modal histograms and further calculates the optimal threshold, giving rise to the above two classes in such a way that their joint distribution is negligible. This taken up to more levels leads to Multi Otsu method [6]. This is an essential part of procedure, which helps to make the algorithm more efficient and quick. Only those features which directly mark the territories for possible presence of tumor cells alone will be extracted from the images.

\subsection{Classification via Machine Learning}

The Support Vector Machine is the neural network employed to perform the classification process as, SVM classifier is outstandingly good for brain tumour detection [7]. Using the features extracted during the preceding development period, the SVM is trained for a broad data collection [8]. Having to reconsider the situation that might arise wherein the data under analysis has 2 individual and distinct classes, Support Vector Machine (SVM) should be the choice undertaken [9]. We use a collection of new brain images of the MRIs is shown in Figure 2. According to SVM technique, most applicable hyper plane is selected in such a way that it lets go of entire set of data dots, belonging to a single class, from the other class.

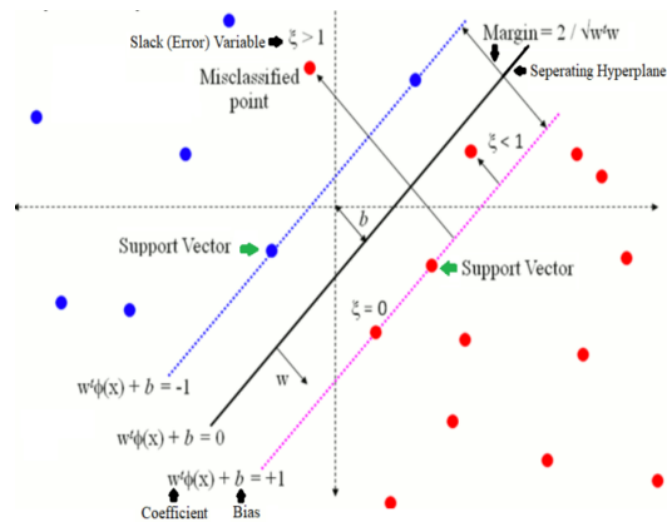

Figure 2. Support Vector Machine

\subsection{Accuracy Features}

Radial Basis Function Accuracy- For data that is dispersed in all dimensions, interpolation of the radial basis function can be very efficient [10-16]. RBFs are capable of offering exceedingly precise numerical resolutions for a wide range of classes.

Linear Accuracy - When it comes to employing linear motion, accuracy and replicability are two features that have to be taken into account. For this a detailed account of the element causing the linear motion and its parameters must be taken into consideration.

Polygonal Accuracy - Instead of employing pixel dots, polygon structures can be used for data for drilling and testing the algorithms. The polygonal data can be transformed into raster standards, which in turn should be within the limits of the features. Across each individual polygon structure the resident accuracy measures can be obtained. 
Quadratic Accuracy -m For medical image analysis, the quadratic accuracy measures proved to be more precise than the linear interpolation based accuracy. Rationalizing the numerator of the quadratic equation provides certain level of precision.

\section{Experimental Results}

To train the computational algorithm, a sufficiently large dataset of the MRI brian images is used. The algorithm is being implemented in version MATLAB 2017. To train the computational algorithm for effective classification a discerning matrix is created in Figure 3 to Figure 5. Classification, most essential parameters like sensitivity, specificity and accuracy were premeditated using following carefully designated formulas which constitute the decision matrix

- TP- True Positive: this involves the case where affected brain is accurately recognized as affected.

- TN- True Negative: this involves the case where normal brain is properly recognized as being normal and unaffected.

- FP - False Positive: this involves the case normal unaffected brain is wrongly classified as affected by tumor brain.

- FN- False negatives: this involves the case brain affected by tumor, whether benign or malignant has been wrongly classified as normal and unaffected.

$$
\begin{aligned}
& \text { Sensitivity }=T P /(T P+F N)(* 100 \%) \\
& \text { Specificity }=T N /(T N+F P)(* 100 \%) \\
& \text { Accuracy }=(T P+T N) /(T P+T N+F P+F N)(* 100 \%)
\end{aligned}
$$

These are the parameters chiefly employed to analyse the classifiers efficiency in terms of performance in Table 1.

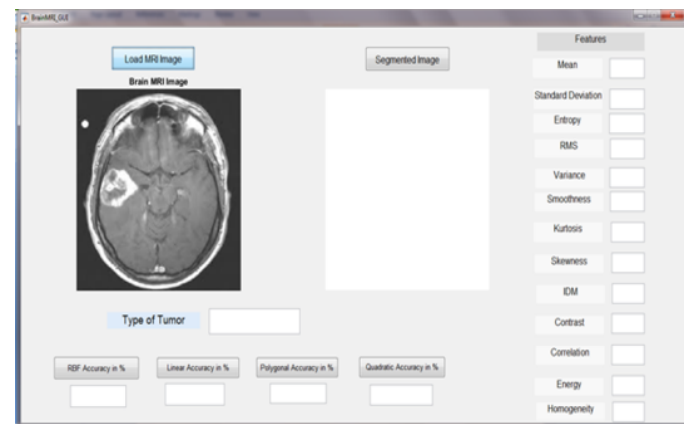

Figure 3. MRI Image Loaded in to GUI

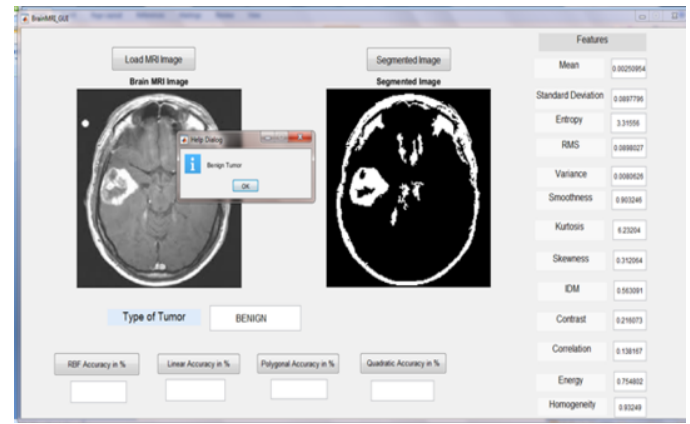

Figure 4. MRI image Segmentation using OTSU \& Classification 


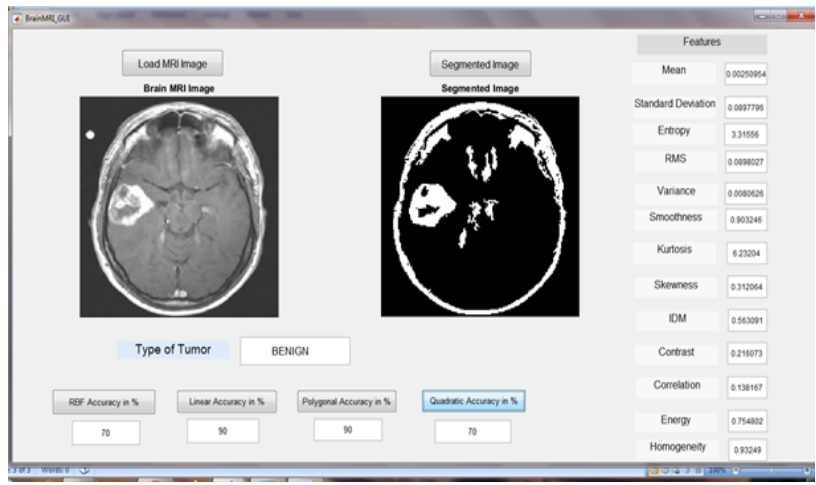

Figure 5. Accuracy Calculation

Table 1. SVM Classifier outputs

\begin{tabular}{|l|l|l|l|}
\hline Kernel Function & Accuracy & Sensitivity & Specificity \\
\hline Linear & $90.87 \%$ & $84.43 \%$ & $98.9 \%$ \\
\hline Quadratic & $85.43 \%$ & $74.33 \%$ & $99.2 \%$ \\
\hline Polynomial & $86.5 \%$ & $76.23 \%$ & $99.39 \%$ \\
\hline
\end{tabular}

\section{Conclusion}

Brain MRI images of this new method have proven to be an effective means of identifying brain tumour. The hybrid methodology of combining contemporary Otsu with Sauvola algorithm proved to provide better segmentation of areas of interest, in the images. Support vector machine for classification provides accurate results for the identification of the brain tumour. The result proves that with precise training input SVMs can distinguish between tumour spots and fittingly categorize them as a benign tumour, malignant tumour or healthy brain tissue. Computational advantages for SVMs are noteworthy. In future research, various data mining modules could be employed for training various kernel functions. This would greatly lift up the efficiency of classifier, and more number of dataset images can be employed for training and testing purposes.

\section{References}

[1] Tustison, N. J., Avants, B. B., Cook, P. A., Zheng, Y., Egan, A., Yushkevich, P. A., \& Gee, J. C. (2010). N4ITK: Improved N3 bias correction. IEEE Transactions on Medical Imaging, 29(6), 1310 1320. https://doi.org/10.1109/TMI.2010.2046908

[2] Shah, M., Xiao, Y., Subbanna, N., Francis, S., Arnold, D. L., Collins, D. L., \& Arbel, T. (2011). Evaluating intensity normalization on MRIs of human brain with multiple sclerosis. Medical Image Analysis, 15(2), 267-282. https://doi.org/10.1016/j.media.2010.12.003

[3] Nyúl, L. G., Udupa, J. K., \& Zhang, X. (2000). New variants of a method of MRI scale standardization. IEEE Transactions on Medical Imaging, 19(2), 143-150. https://doi.org/10.1109/42.836373

[4] Nyú, L. G., \& Udupa, J. K. (1999). On standardizing the MR image intensity scale. Magnetic Resonance in Medicine, 42(6), 1072-1081. https://doi.org/10.1002/(SICI)15222594(199912)42:6<1072::AID-MRM11>3.0.CO;2-M

[5] Zhu, N., Wang, G., Yang, G., \& Dai, W. (2009). A fast 2D otsu thresholding algorithm based on improved histogram. In Proceedings of the 2009 Chinese Conference on Pattern Recognition, CCPR 
2009, and the 1st CJK Joint Workshop on Pattern Recognition, CJKPR (pp. 319-323). https://doi.org/10.1109/CCPR.2009.5344078

[6] LIU Jian-zhuang, Li Wen-qing, "The Automatic threshold of gray level pictures via Two-dimentional Otsu Method", Acta Automatic Sinica,1993.

[7] Haralick, R. M., Dinstein, I., \& Shanmugam, K. (1973). Textural Features for Image Classification. IEEE Transactions on Systems, Man and Cybernetics, SMC-3(6), 610-621. https://doi.org/10.1109/TSMC.1973.4309314

[8] Srivastava, D. K., \& Bhambhu, L. (2010). Data classification using support vector machine. Journal of Theoretical and Applied Information Technology, 12(1), 1-7.

[9] Himanshu makkar and Aditya Pundir, "Image Analysis using improved Otsu's thresholding method", Int. Journal on Recent and Innovation Trends in Computing andCommunication, pp. 2122-2126.

[10] Narayanan, K. L., \& Ramesh, G. P. (2017). VLSI architecture for multi-band wavelet transform based Image compression and Image reconstruction. Journal of Engineering and Applied Sciences, 12(Specialissue2), 6281-6285. https://doi.org/10.3923/jeasci.2017.6281.6285

[11] K.Lakshmi Narayanan, Dr.G.P.Ramesh "Image Compression using Frequency Band Suppression in VLSI Design based Discrete Wavelet Transform" in Int. Journalof Control Theory and Application, 10 (36), 133-139, 2017.

[12] Niranjana.R, Francy Irudaya Rani.E, Manoj P, Naveen Nagarajan S, Raaja P, "Analysis And Detection Of WBC Cancer Cells Using Random Forest Classifier",Int. Journalof Emerging Technology and Innovative Engineering, vol A5, Issue 9, September 2019 (ISSN: 2394 - 6598)

[13] Francy Irudaya Rani. E, Niranjana. R, Abirami.M, Grace Priya.A, Indiranatchiyar.A "Brain Tumor Detection Using Ann Classifier", Int.Journal of Emerging Technology and Innovative Engineering, vol 5, Issue 9, September 2019 (ISSN: $2394-6598$ )

[14] E.Francy Irudaya Rani, S.Ramu, T.Nisha, S.Kavi Sundari, "Predicting a Superlative Classifier for the Identification of Blood Cancer in Human", Int. Journal of Advanced Research Trends in Engineering and Technology (IJARTET), vol. 7, Issue 9, September 2020

[15] E.Francy Irudaya Rani, M.Srimathidevi, E.Sherina, G.Sivasankari, M.Saranya, "A Competent Algorithm for the Detailed Analysis of Skin Images from Dermoscopy", Int. Journal of Advanced Research Trends in Engineering and Technology (IJARTET), vol. 7, Issue 9, September 2020.

[16] Lakshminarayanan, K., Muthukumaran, N., Robinson, Y. H., Shanmuganathan, V., Kadry, S., \& Nam, Y. (2021). Deep Learning-Based Hookworm Detection in Wireless Capsule Endoscopic Image Using AdaBoost Classifier. Computers, Materials and Continua, 67(3), 3045-3055. https://doi.org/10.32604/cmc.2021.014370 